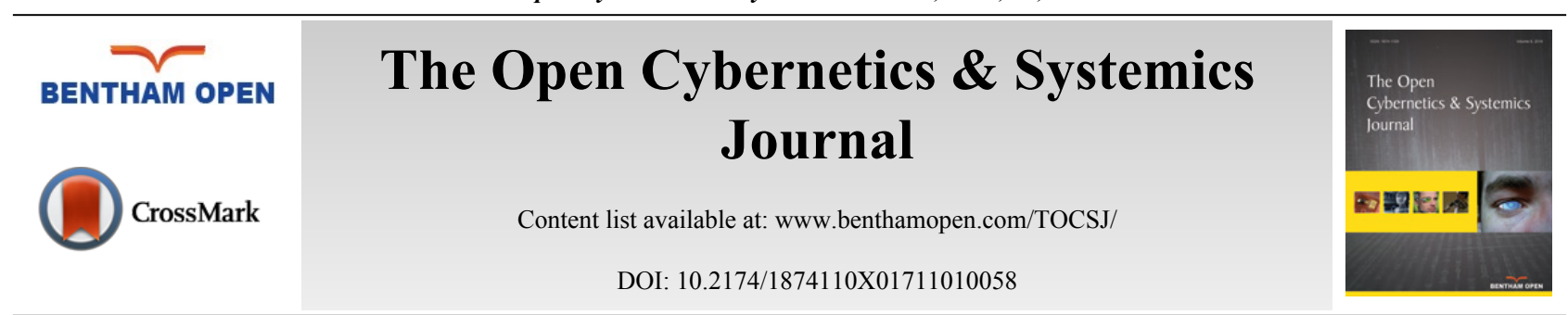

RESEARCH ARTICLE

\title{
Palmprint Identification Using Image Reconstruction Based Double DBNs
}

\author{
Xin Pan ${ }^{1, *}$, Dandan Zhao ${ }^{2}$, Tong Chen ${ }^{1}$, Jiangping Liu ${ }^{1}$, Zhihong $\mathrm{Yu}^{3}$ and Heru Xue ${ }^{1}$ \\ ${ }^{I}$ College of Computer and Information Engineering, Inner Mongolia Agricultural University, Huhhot, Inner Mongolia \\ 010018, China \\ ${ }^{2}$ Zhengzhou Tourism College, Zhengzhou, Henan 450009, China \\ ${ }^{3}$ College of Mechanical and Electrical Engineering, Inner Mongolia Agricultural University, Huhhot, Inner Mongolia \\ 010018, China
}

\begin{abstract}
A novel approach of palmprint recognition using image reconstruction based on double DBNs (IR-DDBN) was proposed in this study, as principal component analysis (PCA) ignores the higher order statistics in feature extraction. Three main steps were involved in the algorithm. Firstly, whitening PCA was utilized to extract the prominent characteristics of the original palmprint image. The second step included reconstructing the original image and calculating the residual image for the residual features between the original and reconstructed images. Finally, the double DBNs were used for classification. The experimental results demonstrated better performance of the proposed algorithm by comparing with the traditional algorithms (PCA, LBP, HOG and DBN) with higher recognition rates, especially for relatively small training samples.
\end{abstract}

Key words: Deep learning, Image reconstruction, Double DBNs, Deep belief nets, Whitening PCA.

\section{INTRODUCTION}

Palmprint identification is one of the key technologies in biometrics to identify a person based on palmprint images, containing creases, wrinkles and ridges, with advantages of low intrusion and easy use, etc [1]. A typical recognition system is composed of five parts, including palmprint scanners, preprocessing, feature extraction, matcher and database [2]. As the most important stage, feature extraction has an impact on the identification result directly. The numerous feature extraction techniques of palmprint recognition can be broadly classified into line-based approaches, subspacebased approaches, statistical approaches [2]. Some researches supplemented appearance-based, texture based and hybrid approaches [3]. Principle Component Analysis (PCA) [4] and Independent Components Analysis (ICA) [5] are the classical tools in subspace-based methods. Recently, deep learning was applied in palmprint recognition. Deep scattering convolutional network whose architecture and filters were predefined wavelet transform provided promising result [6]. In our previous work, we also found that the appropriate DBN model can enhance the performance of palmprint recognition in accuracy [7].

PCA, one of the most popular appearance-based techniques, was applied in palmprint identification for Eigenpalm extraction by Lu et al. [4]. Following this, the two-dimensional PCA, and the integration of PCA and other approaches have been proposed to improve the recognition performance [8 - 10]. The above PCA approaches decomposed the feature space orthogonally into principle components and residual components, in which the former parts were chosen as the projection matrix. However, the discarded data of residual space still kept the information for classification in the study of joint space theory [11], and were successfully applied in the reconstruction-based face recognition [12, 13]. Although manifold-based reconstruction [14] and sparse reconstruction [15] both presented better performance to

\footnotetext{
* Address correspondence to this author at the College of Computer and Information Engineering, Inner Mongolia Agricultural University, 306 Road Zhaowuda, Saihan District, Huhhot , Inner Mongolia, China; Tel: 86-15847129078; E-mails: pxffyfx@126.com, xinpan@yahoo.com
} 
capture the reconstruction residual/errors in the images, the calculation procedure is more complicated and timeconsuming than the subspace approach. Moreover, the palmprint images in our study do not involve the works of image occlusion and completion. So PCA reconstruction is efficient and feasible for our research.

Nevertheless, both the PCA principal space and the residual space cannot well solve the problem of training samples in an efficient way. With the decrease of training samples, PCA can not get efficient features for discrimination. Deep learning is a subfield of machine learning which attempts to learn high-level abstractions in data by utilizing hierarchical architectures [16]. It allows computational models that are composed of multiple processing layers to learn representations of data with multiple levels of abstraction [17], which has made achievements in image recognition [18 - 20], voice recognition [21 - 23], natural language processing, etc. Various deep learning models were extensively investigated to enhance the learning performance. Deep learning concept was incorporated into robust information theoretic framework to reduce the uncertainties in general discriminative data representation tasks [24]. A deep transfer metric learning method was as well proposed to learn a set of hierarchical nonlinear transformations for cross-domain visual recognition by transferring discriminative knowledge from the labeled source domain to the unlabeled target domain [25]. It was reported that the concepts of fuzzy learning into deep learning can overcome the shortcomings of fixed representation [26]. Furthermore, Multimodal deep learning was used to train deep networks for a shared representation between modalities and evaluate it on a unique task [27]. A hybrid convolutional network-Restricted Boltzmann Machine (ConvNet- RBM) model was proposed for face verification to learn high-level relational visual features with rich identity similarity information [28].

An unsupervised layered training algorithm based on Deep Belief Network (DBN) was proposed by Hinton in 2006 [29], called as "the third generation of network" [30]. DBN can well simulate layered visual processing system by learning the abstract features of different layers from up-to-down unsupervised learning and bottom-to-top supervised learning without manual selection during in the whole training procedure [29, 31]. Double DBNs in which two individual DBNs were combined in the last layer to eliminate the ambiguity was proposed in an object recognition and pose estimation system [32].

Taking together both procedures, we developed a new algorithm for palmprint recognition using image reconstruction based double DBNs (IR-DDBN), in which the features of the prominent component space and residual space, extracted by the former PCA image reconstruction, were trained by two separated DBNs with unsupervised learning and combined at the last layer for supervised learning and classification. Three main steps were involved in the algorithm. Firstly, whitening PCA method was utilized to extract the principal components characteristics of ROI palmprint image. Secondly, reconstructing the original image and calculating the residual image for the residual features between the original and reconstructed images. Finally, the double DBNs were used for classification.

The rest of this paper is organized as follows: Sections 2 and 3 introduce image reconstruction based on PCA and double DBNs, respectively. Section 4 presents the proposed algorithm based on image reconstruction and double DBNs. Experimental results are given in Section 5.

\section{IMAGE RECONSTRUCTION BASED ON WHITENING PCA}

Different from the traditional PCA, in which the discarded higher-order statistical components still contributes to image classification, image construction based on whitening PCA can utilize both principal features and higher-order features [13]. Generally, image reconstruction based on whitening PCA can be divided into two stages, whitening PCA and image reconstruction.

\subsection{Whitening PCA}

PCA is one of the most commonly used unsupervising learning methods for dimension reduction. Given $\mathrm{n}$ images of $\mathrm{m}$ classes $i_{1}, i_{2}, \ldots \ldots, i_{n}$ (arranged to column vector), the average palm is

$$
u=\frac{1}{\mathrm{n}} \sum_{k=1}^{\mathrm{n}} i_{k}
$$

The corresponding covariance matrix is

$$
C=\frac{1}{n} \sum_{k=1}^{n}\left(i_{k}-u\right)\left(i_{k}-u\right)^{T}
$$


Let $Z=\left[i_{1}-u, i_{2}-u, \ldots \ldots, i_{n}-u\right] \in \mathrm{R}^{\mathrm{n} \times \mathrm{n}}$, then the covariance matrix $C$ is

$$
C=\frac{1}{n} Z Z^{T}
$$

The eigenvalues of $C$ can be sorted in a descending order, and the eigenvectors corresponding to the former eigenvalues $\left[\lambda_{1}, \lambda_{2}, \ldots \ldots . ., \lambda_{r}\right]$ are chosen when the dimension of transformed data is required to reduced to $\mathrm{r}$-dimension. Generally, $\boldsymbol{W}_{n \times r}$ composed of the projection vectors $W_{1}, W_{2}, \ldots \ldots$, and $W_{r}$ is referred to as projecting matrix. Although PCA can reduce the dimension of the data effectively; the relevance of the neighborhood cannot be changed. Regulation the extracted feature by whitening can not only keep the same covariance of all the features, but also reduce the relevance of the data [13].

Suppose $R=\operatorname{diag}\left[\lambda_{1}, \lambda_{2}, \ldots \ldots . ., \lambda_{r}\right]$, the corresponding transform matrix $W=\left[W_{1}, W_{2}, \ldots \ldots, W_{r}\right]$, whitening matrix $W_{w}=W \cdot R^{-1 / 2}$, then the whitening process can be represented as follows [13]

$$
y=W_{w}^{T} \bullet(x-u)
$$

Where $x$ and $y$ correspond to a given image and the whitened image, respectively.

\subsection{Image Reconstruction}

The reconstruction of $x$ can be obtained according to Eq.4 in Section 2.1 as follows [13]:

$$
\overline{\bar{x}}=W_{w} \bullet y+u
$$

The residue error is the difference of the original image and reconstruction image:

$$
\bar{x}=x-\bar{x}
$$

Figs. (1a, b and c) correspond to a palmprint ROI image, the reconstruction image, and the residue error image (PCA dimension 100), respectively.

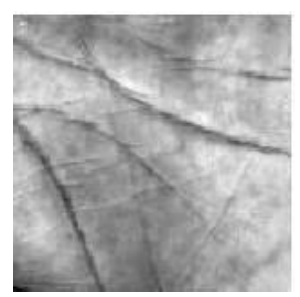

a) ROI image

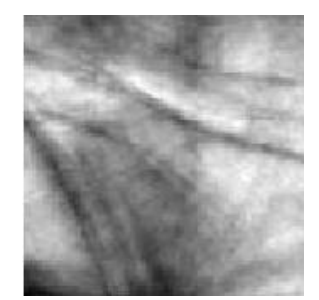

b) Reconstruction image

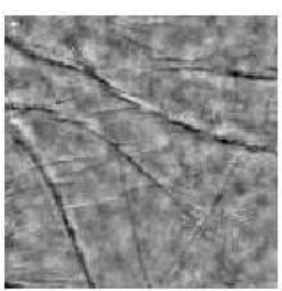

c) Residue error image

Fig. (1). Original palmprint image Fig. (1a), reconstruction palmprint image Fig. (1b) and residue error palmprint image Fig. (1c).

\section{DOUBLE DBNs}

In 2006, Hinton from the University of Toronto proposed an unsupervised training algorithm based on Deep Belief Network (DBN), a probabilistic generative model superimposed by multi-layer Restricted Boltzmann Machine (RBM), for more abstract higher-level representation by arranging the lower-layer features [29 - 31]. The architecture of double DBNs is shown in Fig. (2), where two individual single DBNs can be combined in the last layer for supervised classification, so as to obtain more information of an object [32]. Different kinds of images or features can be trained as the input of visible-layer of $\mathrm{DBN}_{1}$ and $\mathrm{DBN}_{2}$, respectively. In that followed, the output of hidden previous-layer RBM became the input of the next-layer RBM. Finally, the output of the whole DBN was the output of the last-layer RBM, in which a logistic regression layer was superimposed for classification. 


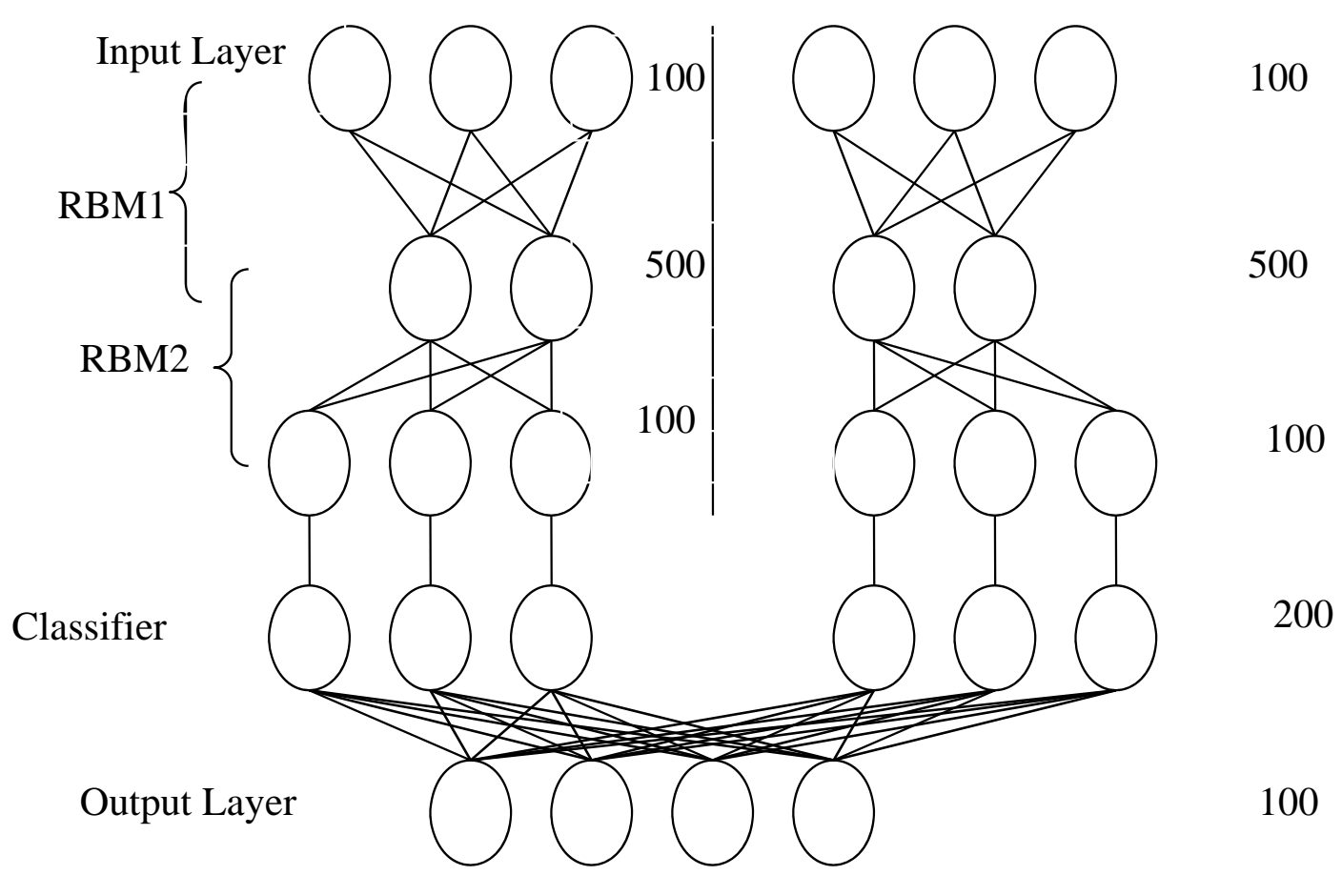

Fig. (2). The architecture of double DBNs.

\section{PALMPRINT RECOGNITION BASED ON IMAGE RECONSTRUCTION AND DOUBLE DBNS}

In order to make full use of residue error of whitening PCA and explore more robust features in deep learning, we proposed a palmprint recognition method using image reconstruction and double DBNs in this paper. Having decomposed the palmprint images onto PCA principal space and residue error space, two individual DBNs were used to train the features, respectively. Then the integration in the last layer for supervised learning to obtain the labels of the input data were obtained by the classification of BP network regression and Softmax regression [31]. Hence, more intrinsic features were trained effectively for palmprint recognition. The flowchart of the algorithm is illustrated as Fig. (3), and accordingly the concrete procedure of the algorithms can be described as follows.

Firstly, whitening PCA method was utilized to extract the principal components characteristics of ROI palmprint image. Palmprint ROI images preprocessed by alignment and normalized to a size of 128x128 were divided into training set and test set. Each training sample was stored in row to constitute the training space. Projection matrix $\mathrm{W}_{1}$ can be obtained by Whitening PCA (see Section 2.1). We set the dimension feature 100, each training sample and test sample can be projected onto the projection matrix to yield feature vector $F_{1}$ and Test $_{1}$, respectively.

Secondly, reconstruct the original image to get the residual features. The residual error can be obtained by calculating the difference between original image and reconstructed image. Then transform the residue error space composed of training samples with whitening PCA to get the projection matrix $\mathrm{W}_{2}$. Project the residue error image of training samples and test samples onto $\mathrm{W}_{2}$ to obtain feature vector $\mathrm{F}_{2}$ and $\mathrm{Test}_{2}$, respectively.

Finally, the double DBNs are applied for classification. Input the two groups of features, Principal Feature $1\left[\mathrm{~F}_{1}\right.$, Test $\left.{ }_{1}\right]$ and Residue Feature $2\left[\mathrm{~F}_{2}, \mathrm{Test}_{2}\right]$ to $\mathrm{DBN}_{1}, \mathrm{DBN}_{2}$ for independent training, respectively, and combine them in the upper-layer for supervised regressive training, and eventually get the recognition results.

\section{EXPERIMENTAL RESULTS}

In order to testify the effectiveness of the algorithm, we conducted 2 groups of experiments. One group was used to test the performance of single DBN and double DBNs with principal components and residue components, the other group was used to compare the performance of the proposed algorithm with other classical approaches. The Beijing Jiaotong University (BJJTU) Palmprint Database contains 3460 palmprint images of $292 \times 413$ pixels of 72 dpi from 346 
palms by a small-scale image scanner, each has 10 samples. The central part of $128 \times 128$ pixels cropped from original palms constitutes a ROI palmprint database (see Fig. (4)). Another Palmprint Database of Hong Kong Polytechnic University (PolyU), available from http://www.comp.polyu.edu.hk/ biometrics, was used to test the robustness of the algorithm. PolyU Palmprint Database contained 8000 palmprint images from 800 palms, and the ROI image size is $128 \times 128$. All the experiments were executed on a computer system of Intel(R)Core(TM) $3.20 \mathrm{GHz}$ and 4GB RAM with Matlab R2011a.

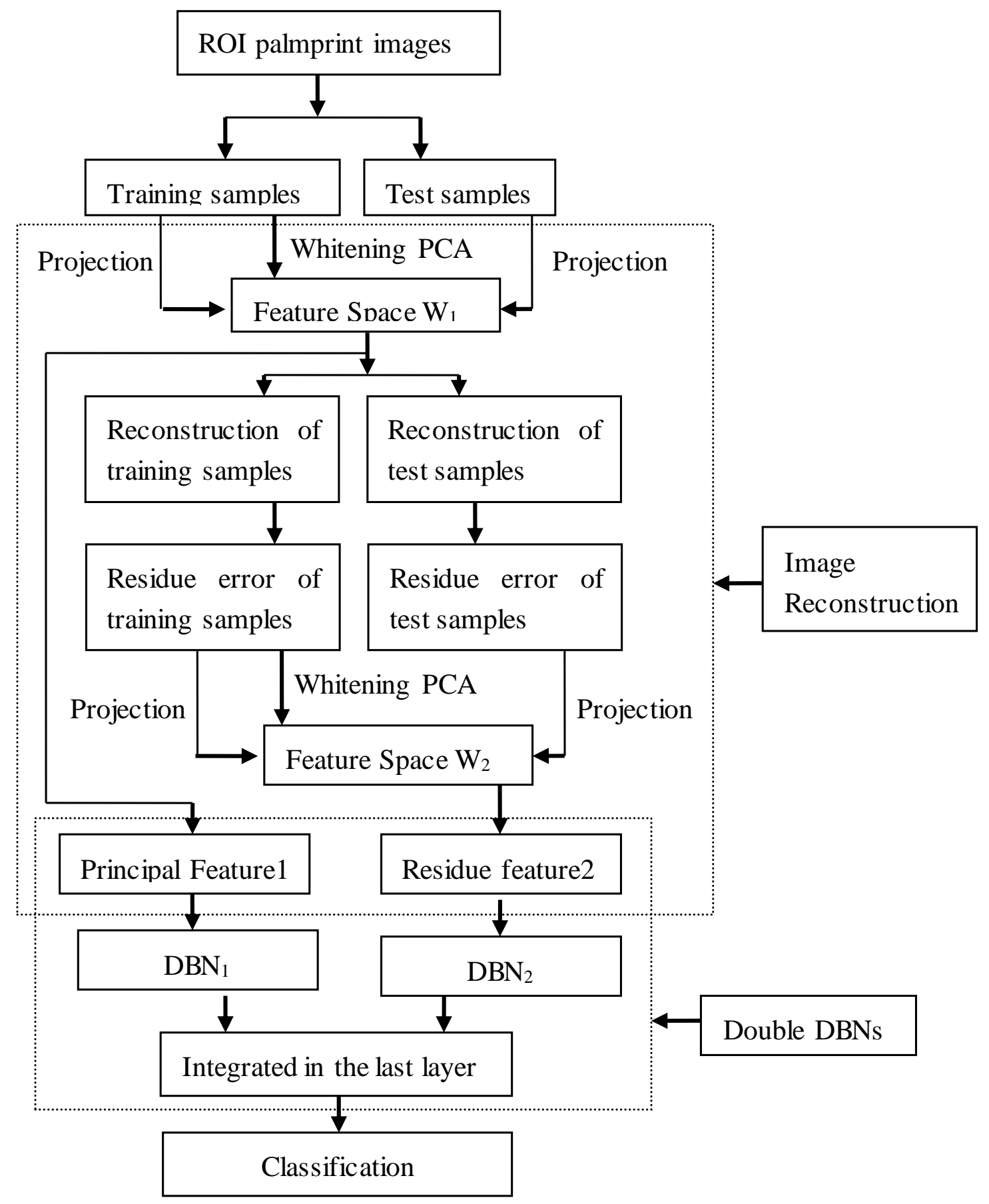

Fig. (3). Flowchart of the proposed algorithm. 


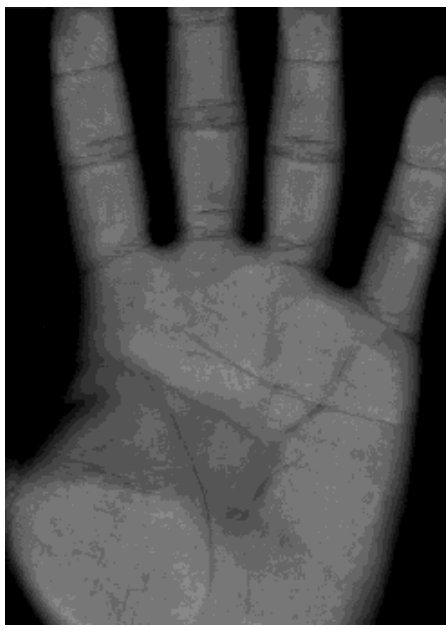

a) Original palmprint image

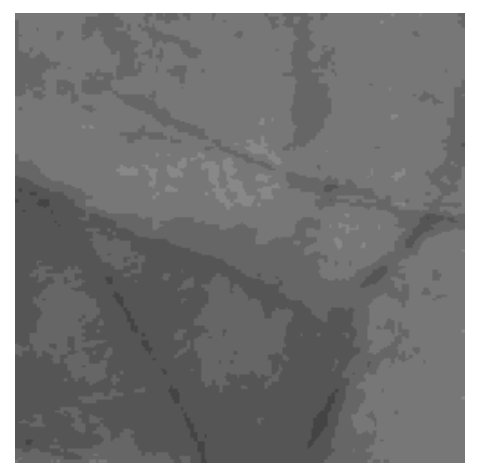

b) ROI image

Fig. (4). Original image and its ROI in BJJTU palmprint database.

\subsection{Comparison of Recognition Performance of Traditional DBN and Double DBNs}

In this group, we compared the running time and recognition accuracy of single DBN with principal components $(\mathrm{DBN}(\mathrm{P}))$ and residue components $(\mathrm{DBN}(\mathrm{R}))$ and image reconstruction based double DBNs (IR-DDBN) taking advantages of both components of whitening PCA. Both DBN and double DBNs consisted of two layers of RBM, and the numbers of DBN and double DBNs were not set too high for an efficient calculation. The numbers of 4 hidden layer units of DBN were 100,500,100, and 100, respectively. The numbers of 4 hidden layer units of double DBNs were 100,500,200, and 100 (Fig. (2)), respectively. We set batchsize 100 in the experiments because at least one image of each class was required in every batch processing [31].The iteration times and learning rate were set 40 and 0.0003 , respectively, according to our previous study [7].

In order to reduce the total running time, 1000 palm images of 100 classes were chosen randomly from BJJTU Palmprint Database in the experiments. The training samples and test samples were both 500, i.e., each class has 5 training samples and 5 test samples, respectively. Owing to the recognition accuracies trained by DBN model varied each time, the average of 10 outputs was used to represent the experimental results. (Table 1) listed the recognition accuracy (\%) and running time(ms) of traditional DBN and double DBNs based on image reconstruction in PCA space and residue error space, where $\mathrm{DBN}(\mathrm{P})$ and $\mathrm{DBN}(\mathrm{R})$ represent the recognition rate of $\mathrm{PCA}$ and residue error space, respectively. $T_{1}, T_{2}$ and $t$ were the training time, test time and total time, respectively.

Table 1. Comparisons of the recognition accuracy (\%) and running time (ms) between single DBN and double DBNs (5 training samples per class, other 5 images per class as test samples, 100 classes BJJTU Palmprint Database).

\begin{tabular}{|c|c|c|c|c|}
\hline & Recognition Accuracy (\%) & $\mathbf{T}_{\mathbf{1}}(\mathbf{m s})$ & $\mathbf{T}_{\mathbf{2}}(\mathbf{m s})$ & $\mathbf{T}(\mathbf{m s )}$ \\
\hline DBN(P) & 92.88 & 13.0433 & 0.0116 & 13.0549 \\
\hline DBN(R) & 48.52 & 20.1200 & 0.0098 & 20.1298 \\
\hline IR-DDBN & $\mathbf{9 5 . 5 6}$ & 22.6392 & 0.0159 & 22.6550 \\
\hline
\end{tabular}

As can be seen from Table 1, the recognition accuracy of DBN(P) was $92.88 \%, 44.36 \%$ higher than that of DBN(R) (48.52\%), suggesting that whitening PCA prominent space extracts most information of the image, on the contrast, residue error space only kept partial discriminant information. The recognition accuracy of double DBNs reached $95.56 \%, 2.37 \%$ higher than that of $\mathrm{DBN}(\mathrm{P})$. The improvement was originated from the integration of PCA space and residue error space, which can not only extract the PCA space feature but also keep the residue error for discrimination. The total time consumption of IR-DDBN (22.6550ms) and DBN(R) $(20.1298 \mathrm{~ms})$ was greater than DBN(P) $(13.0549 \mathrm{~ms})$ because the image reconstruction and second whitening PCA training based on the residue space. Compared with $\mathrm{DBN}(\mathrm{R})$, the total time of IR-DBN based on image reconstruction was slightly longer for more features were trained in the model. 


\subsection{Performance Comparisons with Other Classical Approaches}

We compared the proposed IR-DDBN with some methods, such as PCA, histogram of gradient (HOG) [33, 34], and local binary (LBP) and convolutional neural networks(CNN) [35] using different number of training samples and classes. HOG was proposed for human detection [33], and was applied block based HOG for multispectral palmprint recognition [34]. The feature dimension of PCA was 100, and Euclidean distance and nearest classifier were used for classification. The block size of HOG was $3 \times 3$. The block size of LBP was $2 \times 2$, the histogram of LBP was used for identification. In CNN model (codes available from https:/github.com/ rasmusbergpalm/DeepLearnToolbox), the learning rate, batchsize and epoch iteration times were set 2, 50, and 300, respectively. In the 6 feature maps (size $28 \times 28$ ), each neural cell is connected with the input of $5 \times 5$ neighborhood. The architectures and parameter settings of traditional DBN and IR-DDBNs were the same as those of above experiments in Section 5.1.

(Table 2) listed the identification performance on BJJTU Palmprint Database. The experiments were executed on different classes of 100, 150, and 200 with different numbers of training samples 3,5,7 per class for training, and the rest images per class were used for validation. 100 classes were taken for example, the training set contained 300,500 and 700 images, respectively, then the corresponding test set contained 700, 500 and 300 images, respectively. The corresponding ratios of training/validation were 7/3, 5/5 and 3/7, respectively. As observed from the table, the recognition accuracies of all the approaches increased with the number of training samples. The enhancement was mainly due to more discriminant information learned from more training samples. IR-DDBN outperformed other classical methods and traditional DBN based on prominent components $\mathrm{DBN}(\mathrm{P})$ and residue components $\mathrm{DBN}(\mathrm{R})$, especially when the training samples are 3 and 5. The experimental results suggested that the proposed approach can extract more sufficient information of whitening PCA and residue components when the number of training samples is not abundant. Another factor contributed to the improvement comes from the learning ability of DBN as compared with other shallow learning methods.

Table 2. Comparisons of the recognition accuracy (\%) of different algorithms with different numbers of training sample per class (BJJTU).

\begin{tabular}{|c|c|c|c|c|c|c|c|c|c|}
\hline & \multicolumn{3}{|c|}{100 Classes } & \multicolumn{3}{c|}{ 150 Classes } & \multicolumn{3}{c|}{ 200 Classes } \\
\hline $\begin{array}{c}\text { Training Samples } \\
\text { Per Class }\end{array}$ & $\mathbf{3}$ & $\mathbf{5}$ & $\mathbf{7}$ & $\mathbf{3}$ & $\mathbf{5}$ & $\mathbf{7}$ & $\mathbf{3}$ & $\mathbf{5}$ & $\mathbf{7}$ \\
\hline DBN(P) & 86.97 & 92.88 & 96.10 & 83.10 & 91.13 & 94.58 & 79.79 & 85.76 & 91.63 \\
\hline DBN (R) & 40.47 & 48.52 & 52.63 & 44.70 & 53.03 & 55.47 & 42.89 & 50.94 & 54.95 \\
\hline IR-DDBN & $\mathbf{8 9 . 2 7}$ & $\mathbf{9 5 . 5 6}$ & $\mathbf{9 7 . 8 7}$ & $\mathbf{8 7 . 1 5}$ & $\mathbf{9 4 . 5 9}$ & $\mathbf{9 7 . 1 1}$ & $\mathbf{8 4 . 3 8}$ & $\mathbf{9 1 . 9 6}$ & $\mathbf{9 5 . 9 8}$ \\
\hline CNN & 76.14 & 89.00 & 93.00 & 80.19 & 89.07 & 92.67 & 78.71 & 88.50 & 92.33 \\
\hline PCA & 87.86 & 93.80 & 97.00 & 81.43 & 90.53 & 95.11 & 80.57 & 83.30 & 94.83 \\
\hline HOG & 84.43 & 92.20 & 96.67 & 84.00 & 91.33 & 96.22 & 83.50 & 89.90 & 95.17 \\
\hline LBP & 74.71 & 79.60 & 85.00 & 73.24 & 77.87 & 81.98 & 72.86 & 77.30 & 80.33 \\
\hline
\end{tabular}

With the number of the classes increase from 100 to 150 and 200, the overall recognition accuracies of all approaches decreased. The identification accuracies of IR-DDBN were $89.27 \%, 87.15 \%$, and $84.38 \%$ when the test classes were 100, 150 and 200, using 3 training samples per class. However, the recognition performance of the proposed algorithms was still superior to PCA, HOG, LBP and traditional DBN approaches. LBP, HOG and PCA showed relatively lower recognition rates than the proposed approach, especially when the training samples are 3 and 5, suggesting that DBN can extract the substantive feature from a relatively small number of training samples. Other DBN approaches can not exceed the proposed method for they did not use the whitening PCA principal components and residue components in the same time. The inferior identification performance of CNN as compared with IR-DDBN may come from the complicated optimizing of parameter setting for $\mathrm{CNN}$ requires excessive training to explore. To sum up, the identification performance of the proposed IR-DDBN outweighed the other approaches in the table when the numbers of training samples and test classes varied.

(Table 3) listed the identification performance of IR-DDBN, CNN, PCA, HOG and LBP executed on PolyU Palmprint Database. Higher identification accuracies than those on BJJTU Palmprint Database may come from the image quality acquired in different circumstances. The identification accuracies of IR-DDBN were 99.44\%, 99.25\%, and $98.75 \%$ when the number of classes were 100, 150 and 200, using 3 training samples per class. When the training samples were 5 and 7 per class, the identification accuracies of IR-DDBN exceeded 99\%. The outperformance of IRDDBN as compared with other algorithms on different databases witnessed its robustness in palmprint identification. 
Table 3. Comparisons of the recognition accuracy (\%) of different algorithms with different numbers of training sample per class (PolyU).

\begin{tabular}{|c|c|c|c|c|c|c|c|c|c|}
\hline & \multicolumn{3}{|c|}{100 Classes } & \multicolumn{3}{c|}{ 150 Classes } & \multicolumn{3}{c|}{ 200 Classes } \\
\hline $\begin{array}{c}\text { Training Samples } \\
\text { Per Class }\end{array}$ & $\mathbf{3}$ & $\mathbf{5}$ & $\mathbf{7}$ & $\mathbf{3}$ & $\mathbf{5}$ & $\mathbf{7}$ & $\mathbf{3}$ & $\mathbf{5}$ & $\mathbf{7}$ \\
\hline IR-DDBN & $\mathbf{9 9 . 4 4}$ & $\mathbf{9 9 . 5 2}$ & $\mathbf{1 0 0}$ & $\mathbf{9 9 . 2 5}$ & $\mathbf{9 9 . 5 5}$ & $\mathbf{9 9 . 9 8}$ & $\mathbf{9 8 . 7 5}$ & $\mathbf{9 9 . 3 9}$ & $\mathbf{9 9 . 7 5}$ \\
\hline CNN & 98.14 & 99.10 & 100 & 97.40 & 98.60 & 99.11 & 96.14 & 97.50 & 98.67 \\
\hline PCA & 96.71 & 98.80 & 99.67 & 97.81 & 99.20 & 99.78 & 97.21 & 98.9. & 99.67 \\
\hline HOG & 93.00 & 97.20 & 99.67 & 91.62 & 96.13 & 98.67 & 91.50 & 95.80 & 98.00 \\
\hline LBP & 91.00 & 93.80 & 97.33 & 87.14 & 91.33 & 95.56 & 87.86 & 91.4 & 95.17 \\
\hline
\end{tabular}

\section{CONCLUSION}

In this paper, we proposed an algorithm based on image reconstruction and double DBNs for palmprint recognition. The features extracted by whitening PCA based on original image and residue space were trained by two separated DBNs and integrated in the last layer for classification. The experimental results witnessed the effectiveness of the algorithm, which proved that the double DBNs based on image reconstruction can not only extract the effective features from a relatively small number of training samples, but is also benefited from the relatively complete information from whitening PCA space and residue error space.

\section{CONFLICT OF INTEREST}

The author confirms that this article content has no conflict of interest.

\section{ACKNOWLEDGEMENTS}

This work was supported partly by the National Natural Science Foundation of China under Grant No.61562067,No. 61461041, and No.51365035, the Postdoctoral Science Foundation of China No. 20100480370 and No. 201104179.

\section{REFERENCES}

[1] D. D. Zhang, Palmprint Authentication, Kluwer Academic Publisher: New York, 2004.

[2] A. Kong, D. Zhang, and M. Kamel, "A survey of palmprint recognition", Pattern Recognit., vol. 42, pp. 1408-1418, 2009. [http://dx.doi.org/10.1016/j.patcog.2009.01.018]

[3] R. Raghavendra, and C. Busch, "Texture based features for robust palmprint recognition: a comparative study", EURASIP J. Inf. Secur., vol. 2015, pp. 1-9, 2015.

[http://dx.doi.org/10.1186/s13635-015-0022-z]

[4] G. Lu, D. Zhang, and K. Wang, "Palmprint recognition using eigenpalms features", Pattern Recognit. Lett., vol. 24, pp. 1463-1467, 2003. [http://dx.doi.org/10.1016/S0167-8655(02)00386-0]

[5] L. Shang, D.S. Huang, J.X. Du, and C.H. Zheng, "Palmprint recognition using FastICA algorithm and radial basis probabilistic neural network", Neurocomputing, vol. 69, pp. 1782-1786, 2006.

[http://dx.doi.org/10.1016/j.neucom.2005.11.004]

[6] S. Minaee, and Y. Wang, "Palmprint Recognition Using Deep Scattering Convolutional Network", arXiv preprint arXiv:1603.09027, 2016.

[7] D. Zhao, X. Pan, X. Luo, and X. Gao, "Palmprint recognition based on deep learning", In: International Conference on Wireless, Mobile and Multi-Media, 2015, pp. 214-217.

[http://dx.doi.org/10.1049/cp.2015.0942]

[8] T. Connie, D. Ngo, A. Teoh, and M. Goh, "Palmprint recognition with PCA and ICA", In: Image and Vision Computing New Zealand 2003, Palmerston North, New Zealand, 2003, pp. 232-227.

[9] L. Lin, "Palmprint Identification Using PCA Algorithm and Hierarchical Neural Network", In: Life System Modeling and Intelligent Computing, Springer Berlin Heidelberg, 2010, pp. 618-625.

[http://dx.doi.org/10.1007/978-3-642-15615-1_73]

[10] J. Tao, W. Jiang, Z. Gao, S. Chen, and C. Wang, "Palmprint recognition based on 2-dimension PCA", In: International Conference on Innovative Computing, Information and Control, 2006, pp. 326-330.

[11] X.U. Bin, and M.A. Jin-Wen, "Theoretical Normalization and Generalization of PCA Joint Subspace Model", J. Signal Process., vol. 29, pp. $1638-1643,2013$.

[12] C. Zhou, L. Wang, Q. Zhang, and X. Wei, "Face recognition based on PCA image reconstruction and LDA", Optik - Int. J. Light Electron Optics, vol. 124, pp. 5599-5603, 2013. 
[13] S. Chen, and H. Yin, "Novel feature compensation method for face recognition based on whitening PCA image reconstruction(in Chinese)", Appl. Res. Comput., vol. 32, pp. 2853-2856, 2015.

[14] Y. Deng, Q. Dai, and Z. Zhang, "Graph Laplace for occluded face completion and recognition", In: IEEE Transactions on Image Processing, vol. 20. 2011, pp. 2329-2338.

[15] Y. Deng, Q. Dai, R. Liu, Z. Zhang, and S. Hu, "Low-rank structure learning via nonconvex heuristic recovery", IEEE Trans. Neural Netw. Learn. Syst., vol. 24, no. 3, pp. 383-396, 2013.

[http://dx.doi.org/10.1109/TNNLS.2012.2235082] [PMID: 24808312]

[16] Y. Guo, Y. Liu, A. Oerlemans, S. Lao, S. Wu, and M.S. Lew, "Deep learning for visual understanding: A review", Neurocomputing, vol. 187, pp. 27-48, 2015. [http://dx.doi.org/10.1016/j.neucom.2015.09.116]

[17] Y. LeCun, Y. Bengio, and G. Hinton, "Deep learning", Nature, vol. 521, no. 7553, pp. 436-444, 2015. [http://dx.doi.org/10.1038/nature14539] [PMID: 26017442]

[18] P. Luo, "Hierarchical face parsing via deep learning", In: IEEE Conference on Computer Vision and Pattern Recognition, 2012, pp. $2480-2487$.

[19] H. Lee, C. Ekanadham, and A.Y. Ng, "Sparse deep belief net model for visual area V2", In: Advances in Neural Information Processing Systems, vol. 20. 2008, pp. 873-880.

[20] Q.V. Le, "Building high-level features using large scale unsupervised learning", In: IEEE International Conference on Acoustics, Speech and Signal Processing, 2011, pp. 8595-8598.

[21] M. Osadchy, Y.L. Cun, and M.L. Miller, "Synergistic face detection and pose estimation with energy-based models", J. Machine Learn. Res., vol. 8, pp. 1197-1215, 2007.

[http://dx.doi.org/10.1007/11957959_10]

[22] E.J. Humphrey, J.P. Bello, and Y. Lecun, "Moving beyond feature design: Deep architectures and automatic feature learning in music informatics", In: 13th International Society for Music Information Retrieval Conference (ISMIR), 2012, pp. 403-408.

[23] F. Seide, G. Li, and D. Yu, "Conversational speech transcription using context-dependent deep neural networks", In: Conference of the International Speech Communication Association (INTERSPEECH), 2011, pp. 437-440.

[24] Y. Deng, B. Feng, X. Deng, and R. Wang, "Deep and structured robust information theoretic learning for image analysis", IEEE Trans. Image Process., vol. 25, pp. 4209-4221, 2016.

[25] J. Hu, J. Lu, and Y.P. Tan, "Deep transfer metric learning", In: IEEE Conference on Computer Vision and Pattern Recognition, 2015, pp. $325-333$.

[26] Y. Deng, Z. Ren, Y. Kong, and F. Bao, "A hierarchical fused fuzzy deep neural network for data classification", IEEE Trans. Fuzzy Syst., 2016. [http://dx.doi.org/10.1109/TFUZZ.2016.2574915]

[27] J. Ngiam, A. Khosla, M. Kim, J. Nam, H. Lee, and A.Y. Ng, "Multimodal deep learning", In: International Conference on Machine Learning (ICML), 2011, pp. 689-696.

[28] Y. Sun, X. Wang, and X. Tang, "Hybrid deep learning for face verification", In: IEEE International Conference on Computer Vision, 2013, pp. 1489-1496.

[29] G.E. Hinton, S. Osindero, and Y.W. Teh, "A fast learning algorithm for deep belief nets", Neural Comput., vol. 18, no. 7, pp. 1527-1554, 2006.

[http://dx.doi.org/10.1162/neco.2006.18.7.1527] [PMID: 16764513]

[30] G. Hinton, L. Deng, D. Yu, G.E. Dahl, A. Mohamed, and N. Jaitly, "Deep neural networks for acoustic modeling in speech recognition: The shared views of four research groups", IEEE Signal Process. Mag., vol. 29, pp. 82-97, 2012. [http://dx.doi.org/10.1109/MSP.2012.2205597]

[31] G.E. Hinton, "A practical guide to training restricted boltzmann machines", Momentum, vol. 9, pp. 599-619, 2010.

[32] D. Liang, K. Weng, C. Wang, and G. Liang, "A 3D object recognition and pose estimation system using deep learning method", In: IEEE International Conference on Information Science and Technology, 2014, pp. 401-404. [http://dx.doi.org/10.1109/ICIST.2014.6920502]

[33] N. Dalal, and B. Triggs, "Histograms of Oriented Gradients for Human Detection", In: IEEE Computer Society Conference on Computer Vision \& Pattern Recognition, 2005, pp. 886-893.

[http://dx.doi.org/10.1109/CVPR.2005.177]

[34] D. Hong, W. Liu, J. Su, Z. Pan, and G. Wang, "A novel hierarchical approach for multispectral palmprint recognition", Neurocomputing, vol. 151, pp. 511-521, 2015. [http://dx.doi.org/10.1016/j.neucom.2014.09.013]

[35] A. Krizhevsky, I. Sutskever, and G.E. Hinton, "ImageNet classification with deep convolutional neural networks", In: International Conference on Neural Information Processing Systems, 2012, pp. 1097-1105. 